\title{
Identification and Validation of Autophagy-Related Genes as Potential Biomarkers and Therapeutic Targets in Atrial Fibrillation
}

\author{
Jiao Zhou ${ }^{1,2}$ \\ Yunlong Dong ${ }^{3}$ \\ Xiang Cai $\mathbb{D}^{1,2}$ \\ Hongbo Yang ${ }^{1,2}$ \\ Tao Guo ${ }^{1,2}$ \\ 'Department of Cardiology, Affiliated \\ Cardiovascular Hospital of Kunming \\ Medical University, Kunming, Yunnan, \\ People's Republic of China; ${ }^{2}$ Department \\ of Cardiology, Fuwai Yunnan \\ Cardiovascular Hospital, Kunming, \\ Yunnan, People's Republic of China; \\ ${ }^{3}$ Department of Gastroenterology, The \\ First Affiliated Hospital of Kunming \\ Medical University, Kunming, Yunnan, \\ People's Republic of China
}

Background: Autophagy is an evolutionary conserved important process for the turnover of intracellular substances in eukaryotes and is closely related to the development of atrial fibrillation (AF). The aim of this study is to identify and validate potential autophagy-related genes (ARGs) of AF through bioinformatics analysis and experimental validation.

Methods: We downloaded two data sets from the Gene Expression Omnibus (GEO) database, GSE14975 and GSE31821. After merging the data of the two microarrays, adjusting the batch effect, and integrating the differentially expressed genes (DEGs) with ARGs to obtain differentially expressed autophagy-related genes (DEARGs). Functional and pathway enrichment analyses were carried out based on Gene Ontology (GO) and Kyoto Encyclopedia of Genes and Genomes (KEGG). Use the STRING database to construct a protein-protein interaction (PPI) network. Finally, mRNA expression levels of DEARGs were validated in right atrial tissue samples from AF patients and non-AF controls by qRTPCR.

Results: Through bioinformatics analysis, we finally identified 11 DEARGs (CDKN1A, CXCR4, DIRAS3, HSP90AB1, ITGA3, PRKCD, TP53INP2, DAPK2, IFNG, PTK6, and TNFSF10) in AF using $[\log 2$ (fold change) $>0.5$ and $\mathrm{P}<0.05$. In the pathway enrichment analysis, the most significantly enriched pathway was the autophagy pathway. The results of validation showed that the expression levels of CXCR4, DAPK2, and TNFSF10 corroborating with our computational findings, and the results were statistically significant $(\mathrm{P}<0.05)$.

Conclusion: Our study demonstrates that these 11 potential crucial ARGs, especially CXCR4, DAPK2, and TNFSF10, may be potential biomarkers and therapeutic targets in $\mathrm{AF}$, which will help the personalized treatment of AF patients.

Keywords: autophagy, AF, Gene Expression Omnibus, hub genes

\section{Introduction}

Atrial fibrillation (AF) is the most common clinically sustained cardiac rhythm disorder. There is a high prevalence of AF all over the world, the highest in North America, Europe, China, and Southeast Asia, with approximately 270-360 cases per 100,000 people. $^{1}$ Due to the lack of understanding of the pathogenesis of AF, the current therapies available can only control AF in a short period of time, but cannot cure the disease, which brings a serious burden to the lives of AF patients. The pathogenesis of AF includes atrial remodeling, electrophysiological mechanisms, and the role of the autonomic nervous system, etc. Accumulating evidence has shown that the pathogenesis of AF involves a variety of biological processes,
Correspondence: Tao Guo Department of Cardiology, Affiliated Cardiovascular Hospital of Kunmin Medical University, II 68 Chunrong West Road, Yuhua Street, Chenggong New City, Kunming, Yunnan, People's Republic of China

Tel $+86 \quad 13888988986$

Fax+86 087I 65199777

Email1785875598@qq.com 
including apoptosis, immunoregulation, and autophagy. ${ }^{2-4}$ Among these biological processes, autophagy plays an important role in the development of AF.

Autophagy is the main intracellular degradation system. In the process of autophagy, the cytoplasm is encapsulated in a double-membrane structure of autophagosomes, and autophagosomes fuse with lysosomes to form autophagolysosomes, which are degraded in autophagosomes. ${ }^{5}$ A lot of evidence shows that autophagy response is closely related to the occurrence and development of cardiovascular disease. ${ }^{6-}$

${ }^{9}$ For example, ischemia injury activates autophagy of cardiomyocytes, enabling cardiomyocytes to cope with nutritional stress and improving cell survival rate during ischemia-reperfusion injury. ${ }^{10}$ For coronary heart disease, enhanced autophagy can not only protect the myocardium against ischemia but also prevent the remodeling of the heart after ischemia. ${ }^{11}$ In recent years, there are also some studies on autophagy and AF. ${ }^{12-14}$ Observation of 170 patients in sinus rhythm who had undergone elective coronary artery bypass grafting, Garcia et al found that impaired autophagy plays an important role in the occurrence of postoperative AF. ${ }^{15}$ Yuan et al indicated that there is AMPK-dependent autophagy in the occurrence of $\mathrm{AF}^{4}$ their subsequent research found that autophagy can induce atrial electrical remodeling through ubiquitin-dependent selective degradation of Cav1.2. ${ }^{16}$ However, the understanding of the role of autophagy in the occurrence and development of AF is far from enough.

The purpose of this study is to deeply understand the potential clinical application value of autophagy-related genes (ARGs) in AF through the Gene Expression Omnibus (GEO) database using bioinformatic methods. Although Liu et al have previously conducted bioinformatics analysis of ARGs and AF, they only analyzed one dataset and did not verify the results. ${ }^{17}$ In this study, we analyzed two datasets and verified the finally identified differential ARGs in patients with AF and non-AF individuals, which further improved the reliability of the results.

\section{Materials and Methods}

\section{Data Collection and Preprocessing}

GSE14975 and GSE31821 (10 samples in GSE14975 and 6 samples in GSE31821) were downloaded from GEO (http://www.ncbi.nlm.nih.gov/geo) and the sample platforms used were GPL570 (HG-U133_Plus_2) Affymetrix Human Genome U133 Plus 2.0 Array. ${ }^{18}$ The two data sets were selected because they were both derived from the patient's atrial tissue and the same sequencing platform.

The data were preprocessed as follows: to annotate the data, the probe names were converted into gene names with ActivePerl 5.28.1 software (https://www.activestate. com/products/perl/downloads/). Then we generated a synthetic dataset from GSE14975 and GSE31821 datasets. For the new dataset, eliminate probes without related gene symbols, and calculate the average expression value of those gene symbols with multiple probes. The "sva" package of $\mathrm{R}$ software was used to remove batch-effects for new dataset batch correction.

\section{Screening of Differentially Expressed Genes (DEGs)}

The DEGs were screened with R software limma package version 3.44 .3 (http://bioconductor.org/packages/release/ bioc/html/limma.html) under the criteria of $[\log 2$ (fold change) $]>0.5$ and $\mathrm{P}<0.05$.

\section{Protein-Protein Interaction (PPI) Network and Functional Analysis of DEGs}

PPI networks of DEGs were analyzed using the STRING online tool (STRING database, version 11.0; https://stringdb.org/cgi/input.pl?sessionId=1 fJdSiXTVpOdandinput page show search $=$ on) to further predict protein functional associations and protein-protein interactions. ${ }^{19}$ It might provide insights into the underlying molecular mechanism of the initiation and progression of diseases. An interaction with a confidence score $>0.70$ was considered statistically significant. The specific process of DEGs function and pathway enrichment analysis is consistent with that described by differentially expressed ARGs (DEARGs).

\section{Autophagy-Related Genes}

We obtained a total of 232 ARGs from the Human Autophagy-dedicated Database (HADb, http://autophagy. lu/clustering/index.html), which provides a more detailed list of human genes involved in autophagy.

\section{Identification of DEARGs}

Data were further analyzed by $\mathrm{R}$ software, taking the intersection of DEGs expression profile and 232 ARGs to identify DEARGs. 


\section{Functional and Pathway Enrichment Analysis of DEARGs}

Through Gene Ontology (GO) enrichment analysis, we can comprehensively understand the biological process, cellular component, and molecular function of DEARGs enriched. In this study, the bohao online enrichment tool (http://enrich.shbio.com/index/ga.asp) was utilized to perform GO enrichment analysis on DEARGs. Terms of which $\mathrm{P}<0.05$ were statistically significant.

The identified DEARGs were also analyzed by pathway enrichment in the Kyoto Encyclopedia of Genes and Genomes (KEGG) to find relevant important pathways. ClusterProfiler version 3.16.1 (https://bioconductor.org/packages/release/ bioc/html/clusterProfiler.html) was used to perform KEGG pathway enrichment analysis in R software. ${ }^{20,21}$ Pathways of which $\mathrm{P}<0.05$ were statistically significant.

A detailed flow chart for the specific process of analysis was shown in Figure 1.

\section{AF Patients and Non-AF Controls}

A total of $10 \mathrm{AF}$ patients and 10 non-AF controls were obtained from the Fuwai Yunnan Cardiovascular Hospital between April 2021 and July 2021. This study included patients who underwent thoracotomy due to mitral valve replacement and/or atrial septal defect. Patients with AF (defined as persistent atrial fibrillation lasting more than 7 days) were assigned to AF, while those without AF were assigned to the control group. Patients were excluded if they had thyroid dysfunction and active rheumatism. This study was approved by the Ethics Committee of Fuwai Yunnan Cardiovascular Hospital (Ethical Application Ref: IRB2021BG-006). Written informed consent was obtained from all individual participants included in the study. Right atrial tissue was collected from patients who participated in the study.

\section{RNA Extraction and Quantitative Real-Time PCR (qRT-PCR)}

Total RNA was extracted from right atrial tissue with TRIzol Reagent (Life Technologies, CA, USA). Reverse transcription was conducted using SureScript-First-strandcDNA-synthesis-kit (GeneCopoeia, Guangzhou, China). qPCR was conducted using a BlazeTaq ${ }^{\mathrm{TM}} \mathrm{SYBR}^{\circledR}$ Green qPCR Mix 2.0 kit (GeneCopoeia, Guangzhou, China) following the instructions. The thermocycling conditions were as follows: initial activation at $95^{\circ} \mathrm{C}$ for $30 \mathrm{~s}$, followed by 40 cycles at $95^{\circ} \mathrm{C}$ for $10 \mathrm{~s}, 60^{\circ} \mathrm{C}$ for $20 \mathrm{~s}$ and $72^{\circ} \mathrm{C}$ for $30 \mathrm{~s}$. GAPDH was used as the internal reference for the mRNA for data normalization. The relative

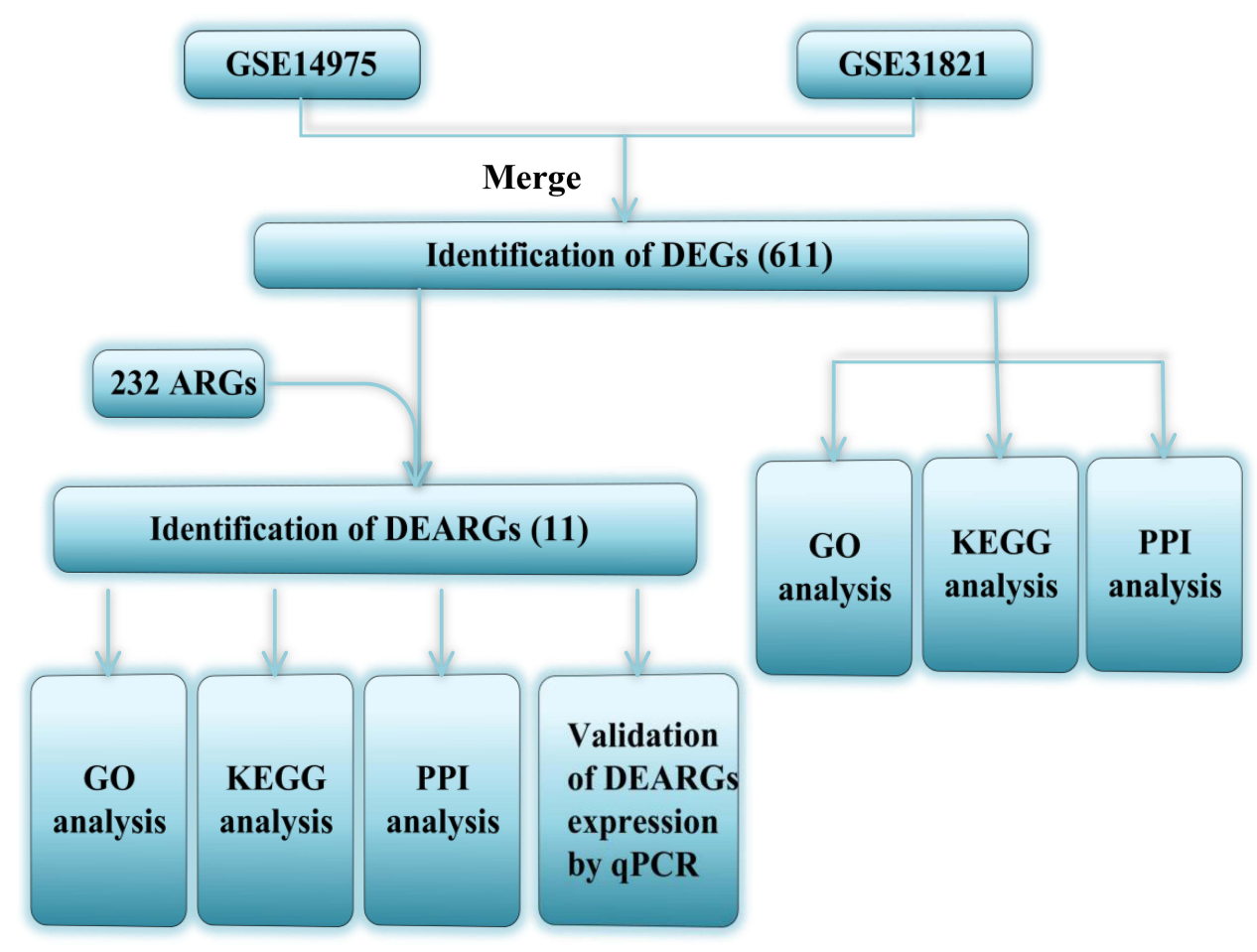

Figure I The flow chart shows the design of the present study.

Abbreviations: DEGs, differentially expressed genes; ARGs, autophagy-related genes; DEARGs, differentially expressed autophagy-related genes; PPI, protein-protein interaction; GO, Gene Ontology; KEGG, Kyoto Encyclopedia of Genes and Genomes. 
Table I Primer Sequences for qRT- PCR

\begin{tabular}{|c|c|c|}
\hline Gene Names & Forward $\left(5^{\prime}-3^{\prime}\right)$ & Reverse (3'-5') \\
\hline CDKNIA & TGAGTTGGGAGGAGGCAG & GAGCGAGGCACAAGGGTA \\
\hline DAPK2 & GAGAGGAGCTGGGGAGTGG & GCTTGATGTGTGGAATGGG \\
\hline DIRAS3 & ACGCCTTCGTCCTGGTCTAC & GGGCATCTGGGATTTCTTCT \\
\hline HSP9OABI & GAACTAAACAAGACCAAGCC & TCCTCAGAGTCAACCACACC \\
\hline IFNG & CATCGTTTTGGGTTCTCTTG & TTTTTCGCTTCCCTGTTTTA \\
\hline ITGA3 & GTAGGAAGCCCCCTCAAG & GGGTAGCCCAGCCATTTA \\
\hline PRKCD & TACGAGATGCTCATTGGC & GTCTTGAAGAAGGGGTGG \\
\hline PTK6 & CGTCTGGTCCTTTGGGATTCTC & GTCGGGTTCTCGTAGCTGGTGA \\
\hline TNFSFIO & AGCAACACATTGTCTTCTCCA & TAAGCTCAAATATTCCСССTT \\
\hline TP53INP2 & AAAGAAAACACAAAGAACGACAA & ACTAAAAAGGCCCCAAAAAAACT \\
\hline CXCR4 & AGCAAGGGTGTGAGTTTGAGA & GAAAGCATAGAGGATGGGGTT \\
\hline GAPDH & CGCTGAGTACGTCGTGGAGTC & GCTGATGATCTTGAGGCTGTTGTC \\
\hline
\end{tabular}

Abbreviation: qRT-PCR, quantitative reverse transcription-quantitative polymerase chain reaction.

expression was calculated by $2^{-\Delta \Delta \mathrm{Ct}}$ method. Primers are available in Table 1.

\section{Statistical Analysis}

The statistical analyses were performed using GraphPad Prism 9.0 software (San Diego, CA, USA). Real-time gene expression levels of our tissue samples were compared using Student's $t$-test. $\mathrm{P}<0.05$ was considered to indicate statistical significance.

\section{Results}

\section{Data Preprocessing and Differential Expression Analysis}

A total of 54,675 probes were obtained from GSE14975 and GSE31821. After preprocessing, 21,644 genes were identified. Considering the criteria for [log2 (fold change)] $>0.5$ and $\mathrm{P}<0.05$, we finally obtained a total of 611 significant DEGs, of which 309 were up-regulated and 302 were down-regulated. The clustering heatmap is shown in Figure 2.

\section{PPI Network and Functional GO Terms and Pathway Enrichment Analyses of DEGs}

Altogether, 202 nodes and 389 interaction pairs were identified in the PPI network (Figure 3). According to the view that highly connected genes were noted to possibly play important roles in diseases, we calculated the connectivity between the nodes through $\mathrm{R}$ software and the results are displayed in Figure 4. Here, the first 6 nodes are all members of the collagen family, including collagen type I alpha 1 chain (COL1A1, degree =21), collagen type III alpha 1 chain (COL3A1, degree $=17$ ), collagen type IV alpha 1 chain (COL4A1, degree $=16$ ), collagen type IV alpha 2 chain (COL4A2, degree =16), collagen type $\mathrm{V}$ alpha 1 chain (COL5A1, degree $=15$ ) and collagen type IX alpha 3 chain (COL9A3, degree $=15$ ) are considering as hub genes in related to AF maintaining.

To investigate the biological effects of DEGs, we performed GO and KEGG functional enrichment analyses, the top $3 \mathrm{GO}$ terms related biological processes were collagen catabolic process (enrich factor: 6.62; P-value: 3.148e-07), collagen fibril organization (enrich factor: 7.92; P-value: $1.713 \mathrm{e}-05$ ) and negative regulation of cellcell adhesion (enrich factor: 7.00; P-value: 3.780e-07), the results are shown in the Figure 5A.

KEGG pathway analysis data appear in Figures $5 \mathrm{~B}$ and C. The results suggest that DEGs were significantly enriched in pathways of protein digestion and absorption (P-value: 4.24e-08), amoebiasis (P-value: 4.72e-06), and IL-17 signaling pathway (P-value: 0.00037 ).

\section{Identification of DEARGs}

Using the DEGs identified in the previous step and extracted the expression values of 232 ARGs. A total of 11 DEARGs were identified (see Table 2 and Figure 6A for details), including 7 up-regulated genes (CDKN1A, CXCR4, DIRAS3, HSP90AB1, ITGA3, PRKCD, TP53INP2) and 4 down-regulated genes (DAPK2, IFNG, PTK6, TNFSF10). Besides, a heat map was visualized to show the relative expression pattern of 11 DEARGs between control samples and AF samples (Figure 6B). 


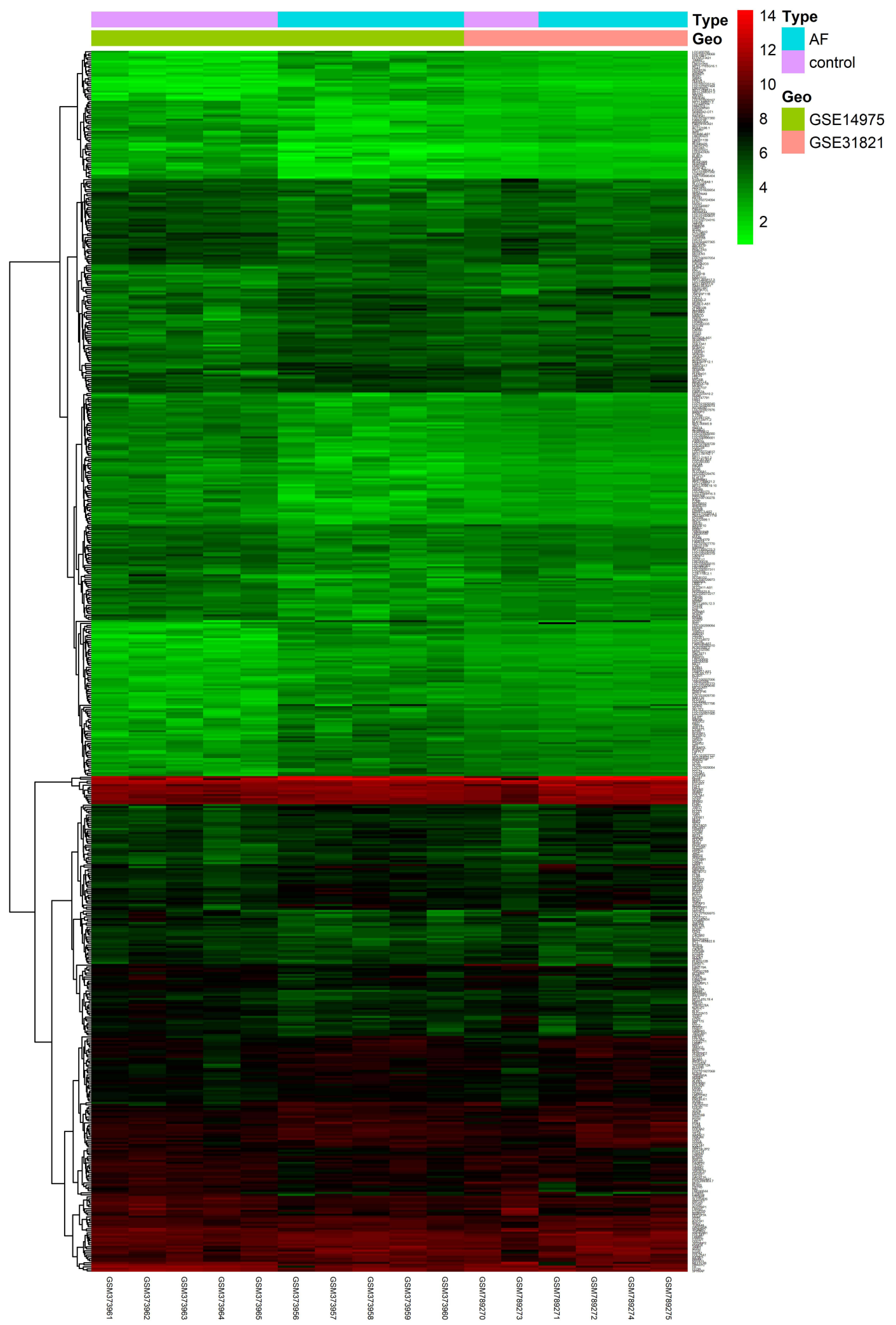

Figure 2 Clustering heatmap of DEGs in GSEI4975 and GSE3 I82I datasets. $n=6$ I I DEGs. The red signifies upregulation, whereas the green indicates the downregulation of genes.

Abbreviation: DEGs, differentially expressed genes. 


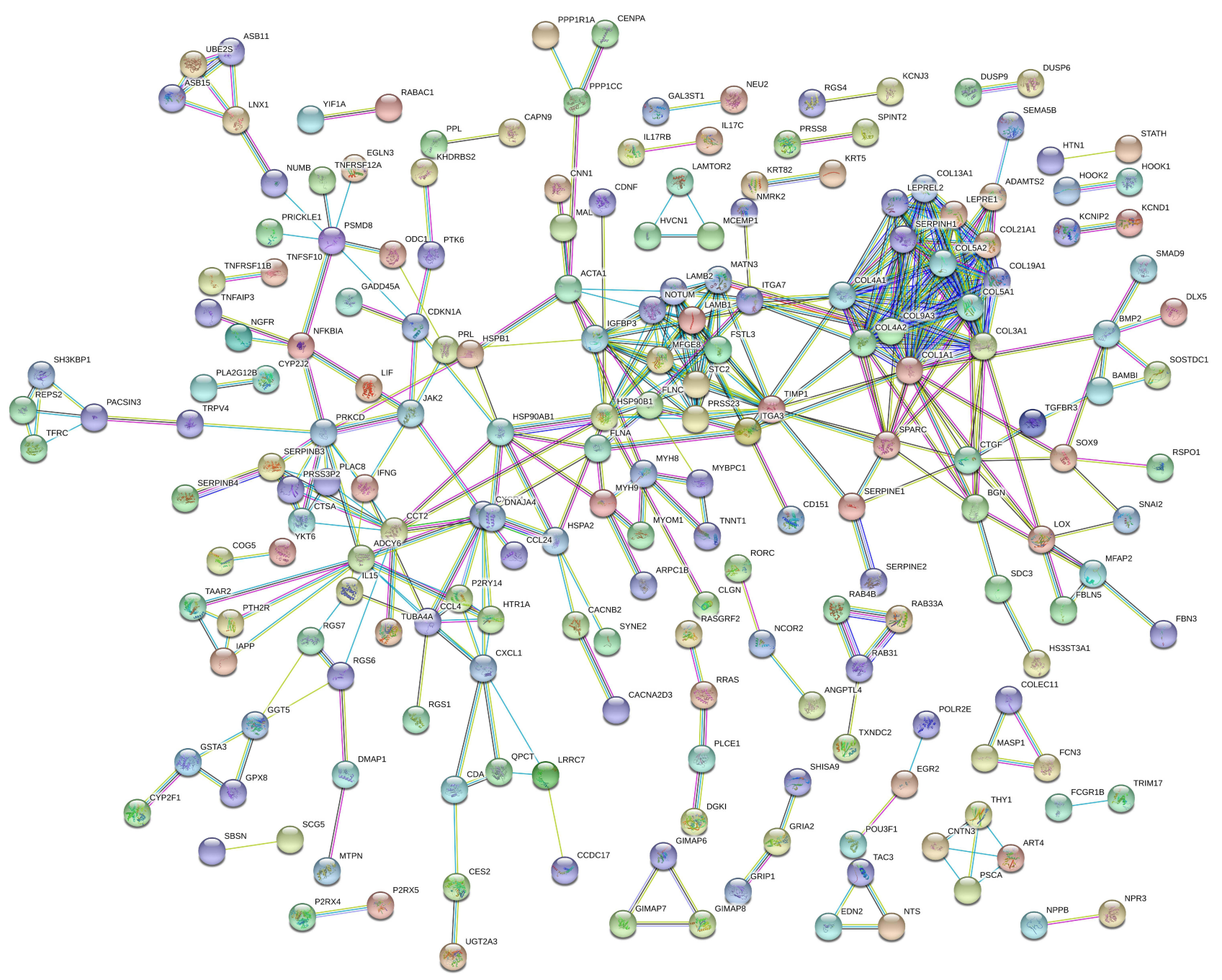

Figure 3 PPI network of DEGs between control samples and atrial fibrillation samples. Abbreviations: PPI, protein-protein interaction; DEGs, differentially expressed genes.

\section{PPI Network and Functional Enrichment of the DEARGs}

The PPI network based on DEARGs consisted of 17 nodes and 90 interaction pairs (Figures 7 and 8 ). The top 8 nodes are mostly proteins related to cell cycle regulation, respectively TP53, CDK4, CDK6, CDKN1A, CCND1, CDK2, CCNA2, and CCNE1.

To further explore the biological functions of the DEARGs, functional enrichment and pathway analyses were performed and the results are presented in Figure 9A-C.

GO enrichment showed that changes in the biological process (BP) of DEARGs were mainly enriched in the protein phosphorylation, protein kinase activation, and apoptotic signaling pathway. (Figure 9A, Table 3).
The results of KEGG enrichment analysis showed that pathways of DEARGs mainly involve pathways in autophagy, necroptosis, and bladder cancer (Figures 9B and C). It is worth mentioning that it is directly enriched into autophagy, which proves the correctness of the analysis results.

\section{Validation of DEARGs Expression in AF Patients and Non-AF Controls}

To confirm the reliability of the bioinformatics analysis, we validated DEARGs in patients with AF and control by qRT- PCR. The clinicopathological variables of cases and controls are summarized in Table 4. Similar to the results of mRNA microarray in AF tissue samples, the expression level of CXCR4 was found to be significantly up-regulated (Figure 10B), the expression levels of DAPK2 and 


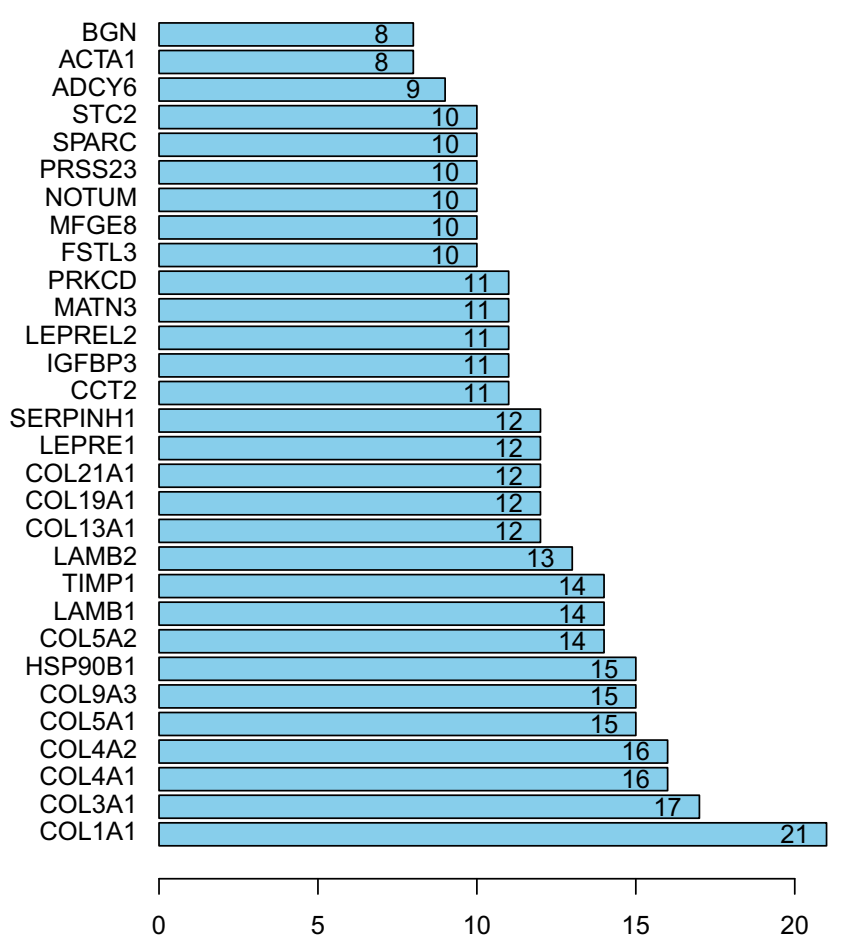

Figure 4 Top 30 nodes of PPI networks of DEGs between control samples and AF samples.

Abbreviations: PPI, protein-protein interaction; DEGs, differentially expressed genes; $A F$, atrial fibrillation.

TNFSF10 were found to be down-regulated (Figures 10F and G). However, the expression levels of CDKN1A, HSP90AB1, and TP53INP2 showed no significant difference between AF patients and non-AF controls (Figure 10A-D). Furthermore, we found that DIRAS3 was significantly down-regulated in $\mathrm{AF}$ patients (Figure 10E), which did not correlate with our bioinformatics analysis. IFNG, ITGA3, PRKCD, and PTK6 were not detected by qRT- PCR due to their low expression levels.

\section{Discussion}

The pathogenesis of AF includes atrial remodeling, electrophysiological mechanisms, and the role of the autonomic nervous system, etc. Among all of them, atrial remodeling is most closely associated with AF. It is well-known that myocardial fibrosis is the most important part of atrial remodeling. When the content of fiber components (collagen fiber) in myocardial tissue is higher, AF is more likely to persist. In this study, PPI network and GO analysis of DEGs also showed that collagen family and collagen catabolic process play a key role in the pathogenesis of AF, especially type I and III collagen, have been confirmed that their metabolic changes are significantly related to $\mathrm{AF}^{22}$ With the in-depth research in recent years, increasing evidence has demonstrated that autophagy may be involved in the regulation of myocardial fibrosis. ${ }^{23-25}$ Chikusetsu saponin IVa can activate autophagy through AMPK/mTOR/ULK1 pathway to reduce isoproterenol-

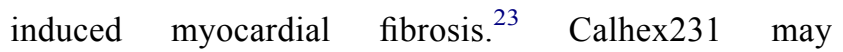
ameliorate myocardial fibrosis by inhibiting autophagymediated activation of NLRP3 inflammasome. ${ }^{26}$ However, extensive validation is needed to improve our understanding of autophagy in the pathogenesis of AF.

Autophagy is strongly linked to the development of $\mathrm{AF}$, but the mechanisms involved are complex. The study of $\mathrm{Hu}$ et al has directly pointed out that, through in vivo and in vitro studies, they found that quercetin can inhibit the expression of miR-223-3p, while enhancing the expression of FOXO3 and activating the autophagy pathway, which significantly inhibits Myocardial fibrosis in $\mathrm{AF}^{27}$ But, there have been no studies using bioinformatics to explore the role of ARGs in AF and to conduct experimental verification. In the present study, we used bioinformatics tools to analyze the integrated data of gene expression profiles from two GEO datasets to identify key ARGs related to the therapeutic targets of AF patients. We found that 11 ARGs (CDKN1A, CXCR4, DIRAS3, HSP90AB1, ITGA3, PRKCD, TP53INP2, DAPK2, IFNG, PTK6, TNFSF10) under the criteria of [ $\log 2$ (fold change)] $>0.5$ and $\mathrm{P}<0.05$ were differentially expressed in $\mathrm{AF}$ patient myocardial tissue samples. Some of these ARGs of AF have been previously studied. For example, the expression of CXCR4 is upregulated in patients with chronic $\mathrm{AF}$, leading to atrial remodeling. ${ }^{28}$ This result is consistent with the results of our bioinformatics analysis and experimental verification. We intend to explore more potential ARGs of $\mathrm{AF}$ in the future.

The STRING database was used to identify the eight nodes with the greatest degree of network connection (TP53, CDK4, CDK6, CDKN1A, CCND1, CDK2, CCNA2, and CCNE1). These targets are mainly regulatory factors related to inflammation, DNA replication and repair, cellular senescence, cell cycle, and so forth. ${ }^{29-32}$ Myocardial fibrosis is one of the important mechanisms for the occurrence of $\mathrm{AF}$, and these proteins may indirectly participate in the regulation of AF through myocardial fibrosis. The greatest number 
A

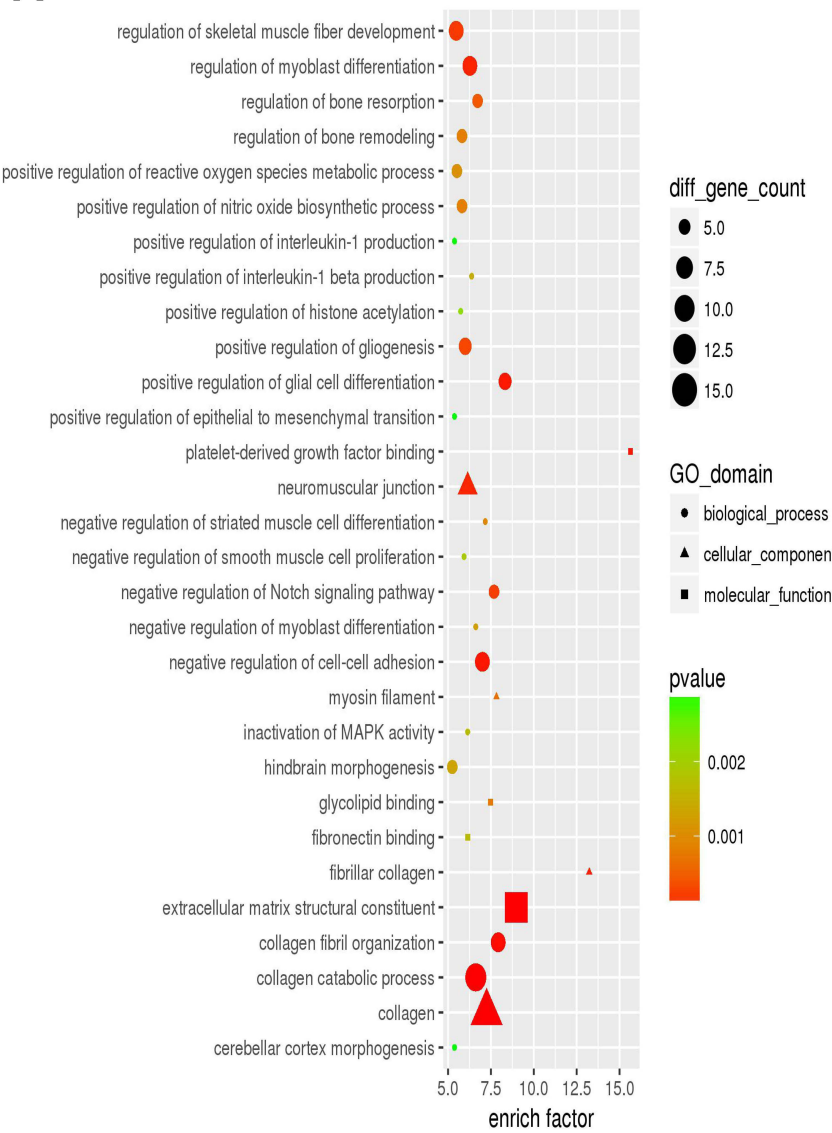

B
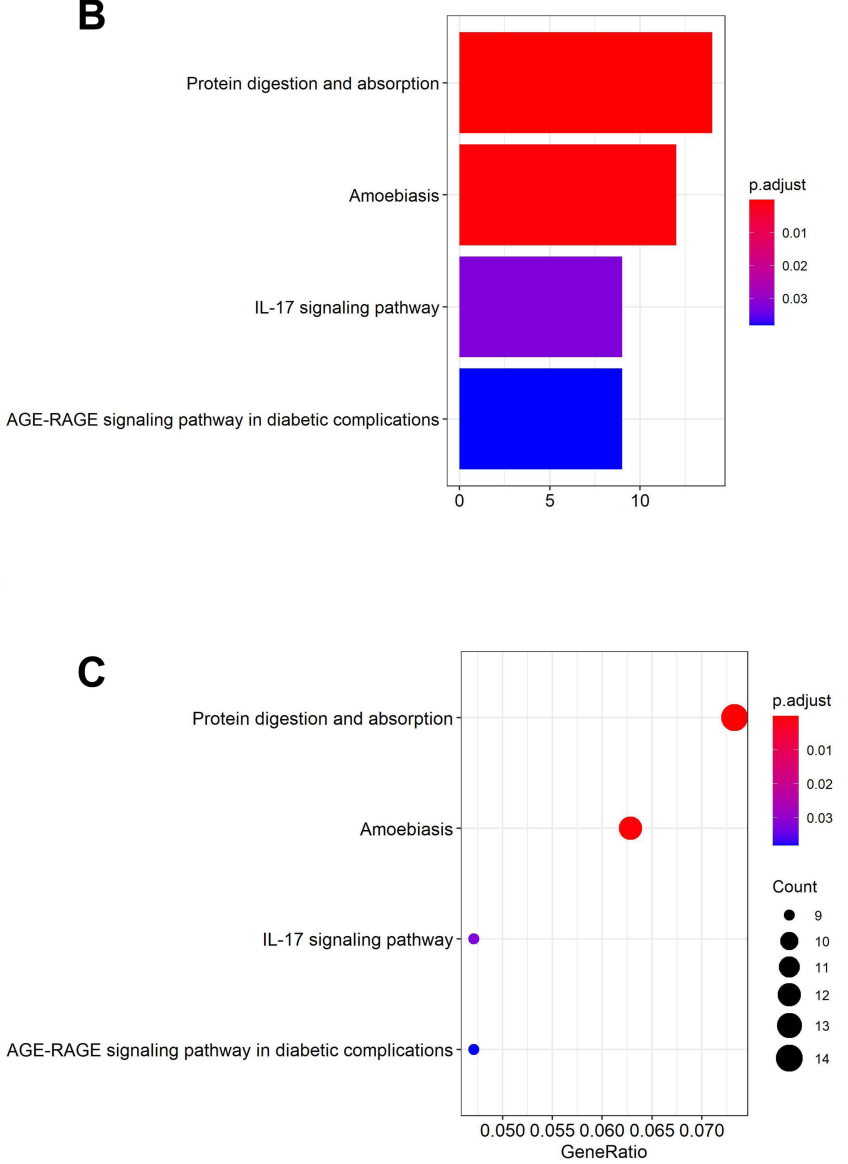

Figure 5 GO (A) analysis shows the biological processes, cellular components, and molecular functions involved in DEGs. Bar plot (B) and dot plot (C) show KEGG pathway enrichment of DEGs.

Abbreviations: GO, Gene Ontology; DEGs, differentially expressed genes; KEGG, Kyoto Encyclopedia of Genes and Genomes.

of node networks ( $\mathrm{n}=20$ ) was TP53. Tumor protein $\mathrm{p} 53$ (TP53) is a critical protein involved in the process of cell cycle, apoptosis, senescence, and DNA repair.
Overactivation of TP53 pathway in cardiomyocytes induces myocardial fibrosis. ${ }^{33}$ Similar to the studies conducted by Chen et al, the TP53 signaling pathway

Table 2 The DEARGs of Merged Data of 6 I I DEGs and 232 ARGs with the Use of Criteria of Log2 (Fold Change) $>0.5$ and P <0.05

\begin{tabular}{|l|l|l|l|l|l|l|}
\hline Gene Names & Log2 FC & AveExpr & t & P-value & Adj. P. Val \\
\hline CDKNIA & 0.6755 & 8.0689 & 2.7433 & 0.0136 & 0.7170 & B \\
CXCR4 & 0.8774 & 6.3564 & 2.8709 & 0.0104 & 0.6877 & -2.8357 \\
DAPK2 & -0.6586 & 7.1420 & -4.1998 & 0.0006 & 0.4001 & -2.6383 \\
DIRAS3 & 0.8147 & 6.0380 & 2.3157 & 0.0330 & 0.7937 & -0.5576 \\
HSP90ABI & 0.6056 & 9.8253 & 2.3393 & 0.0314 & 0.7870 & -3.4772 \\
IFNG & -0.9761 & 3.2009 & -2.4571 & 0.0247 & 0.7607 & -3.4427 \\
ITGA3 & 0.8263 & 5.0535 & $2.348 I$ & 0.0309 & 0.7870 & -3.2693 \\
PRKCD & 0.9270 & 5.1578 & 2.2153 & 0.0403 & 0.8028 & -3.4300 \\
PTK6 & -0.8182 & 3.8179 & -3.7636 & 0.0015 & 0.5276 & -3.6217 \\
TNFSFI0 & -0.7106 & 8.0994 & -2.5080 & 0.0223 & 0.7607 & -1.2334 \\
TP53INP2 & 0.5637 & 9.7921 & 3.7557 & 0.0015 & 0.5276 & -3.1933 \\
\hline
\end{tabular}

Abbreviations: DEARGs, differentially expressed autophagy-related genes; DEGs, differentially expressed genes; ARGs, autophagy-related genes; log2 FC, log2 (fold change); AveExpr, average expression; adj. P. Val, adjust P-value. 


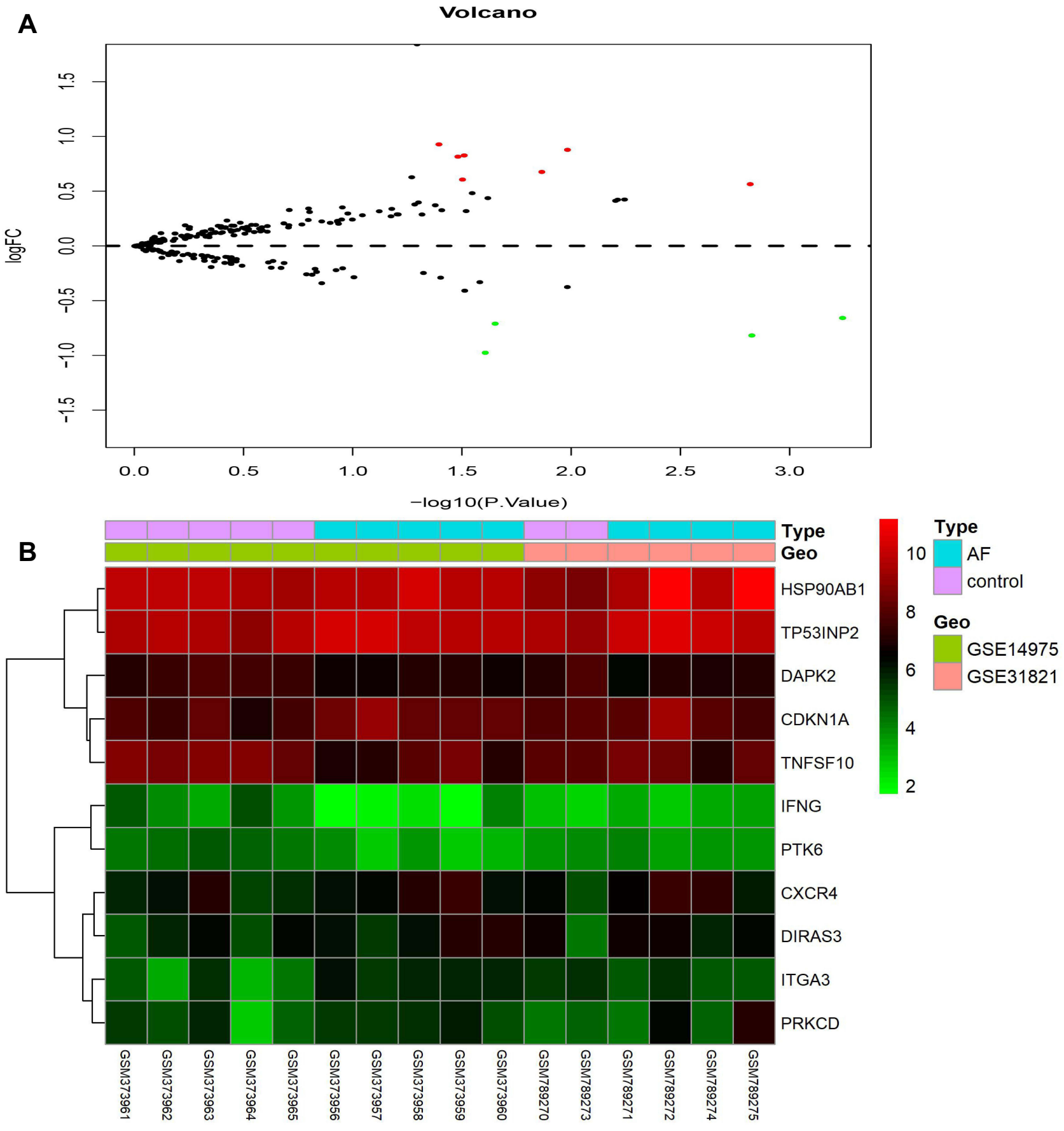

Figure 6 Volcano map (A) shows DEARGs, with red dots representing up-regulated genes, green dots representing down-regulated genes, and the remaining black dots representing no differences gene. Heat map (B) of II DEARGs in AF samples and control samples. Abbreviations: DEARGs, differentially expressed autophagy-related genes; AF, atrial fibrillation.

is activated in ventricular arrhythmias in dilated cardiomyopathy. ${ }^{34}$ Therefore, TP53 may be an important target gene of AF. CDK4, CDK6, CDKN1A, CDK2, and CCNA2 are important proteins involved in the regulation of the mammalian cell cycle. The present study shows that CDK4/6 inhibitors could delay the progression of bleomycin-induced pulmonary fibrosis. ${ }^{35}$ MicroRNA-1 can inhibit the proliferation of cardiac fibroblasts by directly acting on CDK6. ${ }^{36}$ Moreover, activation of AMPK further inhibited CDK2 and cyclin E complexes by up-regulating CDKN1A expression, ultimately suppressing the 


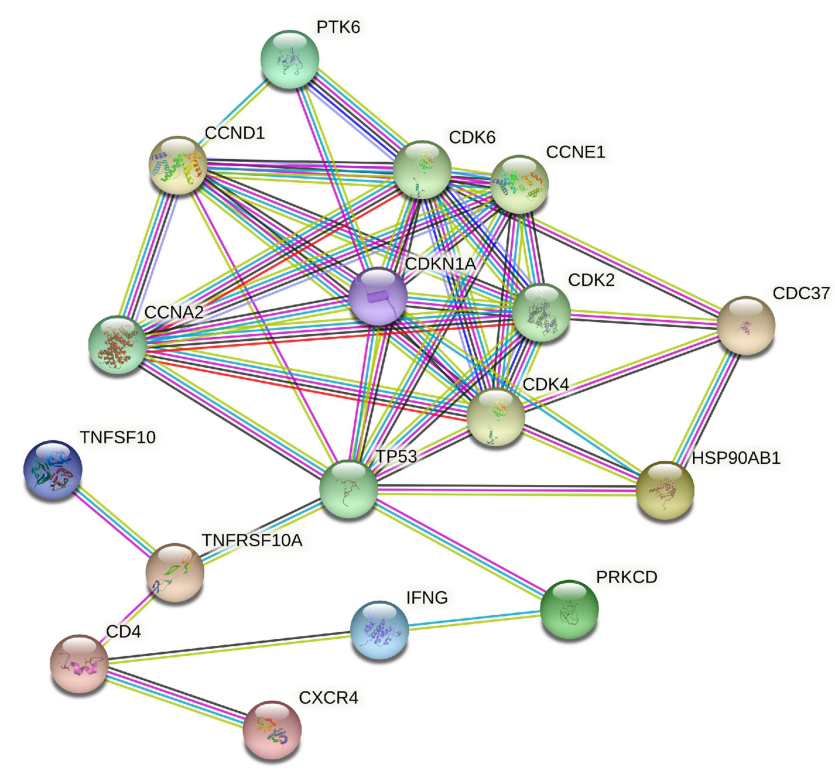

Figure 7 PPI network of DEARGs between control samples and atrial fibrillation samples.

Abbreviations: PPI, protein-protein interaction; DEARGs, differentially expressed autophagy-related genes.

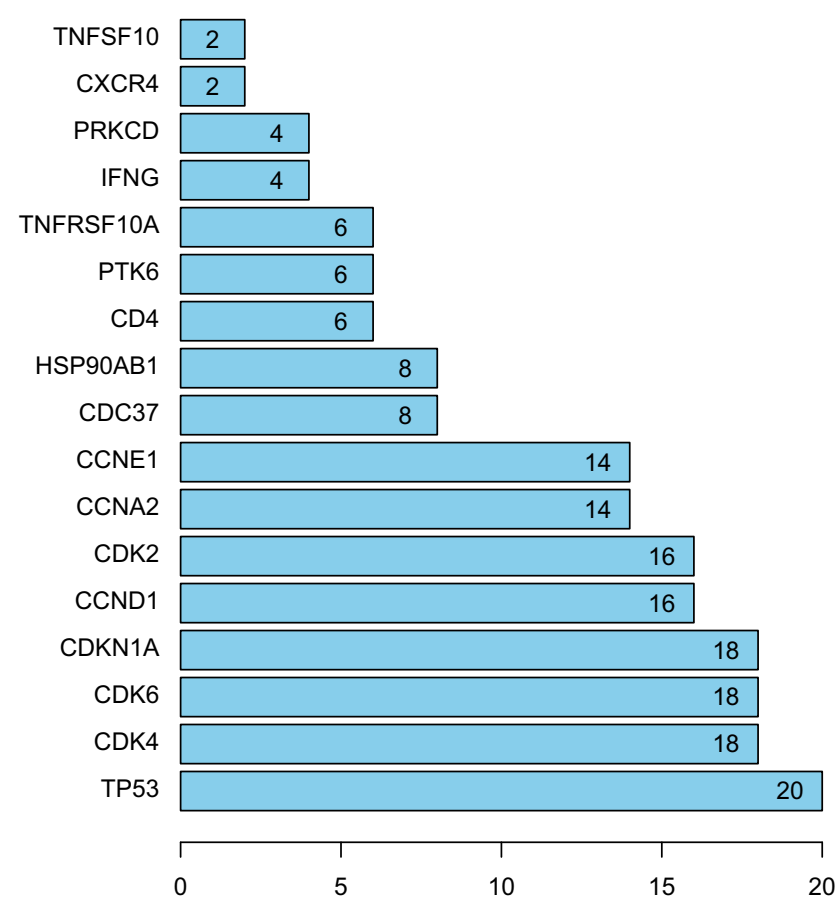

Figure 8 Top 17 nodes of PPI networks of DEARGs between control samples and AF samples.

Abbreviations: PPI, protein-protein interaction; DEARGs, differentially expressed autophagy-related genes; AF, atrial fibrillation.

progression of cardiac fibrosis. ${ }^{37}$ Overexpression of CCNA2 also alleviates myocardial fibrosis in a porcine model of myocardial infarction. ${ }^{38}$ These studies provide a convincing framework for the development of therapies to alleviate myocardial fibrosis based on cardiomyocyte cycle regulation. However, there exists no study to investigate the relationships between CCND1, CCNE1 and myocardial fibrosis.

The potential biological functions of these DEARGs were also analyzed by GO and KEGG enrichment analysis. Functional enrichment analysis in the present study indicated that the GO terms (biological process) were mainly enriched in protein phosphorylation regulation, protein kinase activity, and kinase activity. KEGG pathway enrichment analysis showed that PRKCD, TP53INP2, and DAPK2 were enriched in the autophagy pathway. Some published articles confirmed that autophagy can affect the progress of AF. One paper mentioned that autophagy can promote the occurrence and persistence of AF through the degradation of L-type calcium channels. ${ }^{16}$ One other study found osteopontin induces the activation of AKT/ mTOR, inhibits autophagy, aggravates the AF. ${ }^{13}$ These findings suggest that the study of ARGs and $\mathrm{AF}$ may reveal the pathogenesis of AF.

Based on the bioinformatics analysis results, we further identified the expression level of DEARGs in our clinical samples by qRT-PCR. According to the results of qRT- PCR, the expression levels of CXCR4, DAPK2, and TNFSF10 were in accordance with the bioinformatics analysis results from mRNA microarray. Among them, CXCR4 has been confirmed to be closely associated with the occurrence and maintenance of AF. It was found that CXCR4 expression is up-regulated in patients with chronic AF with mitral valve disease. ${ }^{28}$ Inhibition of CXCL12/CXCR4 axis can reduce the recruitment of inflammatory cells and suppress the hyperactivation of atrial ERK $1 / 2$ and AKT/mTOR signaling pathways, thereby alleviating $\mathrm{AF}^{39}$ As previously mentioned, myocardial fibrosis is one of the important pathogenesis of AF, and CXCR4 also plays a key regulatory role in myocardial fibrosis. It was found that CXCR4 antagonist significantly reduced the expression of collagen I mRNA and alleviated myocardial fibrosis in experimental dilated cardiomyopathy mice. ${ }^{40}$ In addition, study on DAPK2, TNFSF10 and AF is not reported. It should be noted that due to the limited sample volume, we did not conduct experimental verification of differential protein abundance by analyzing gene expression. However, the differential protein identified in this study has also 

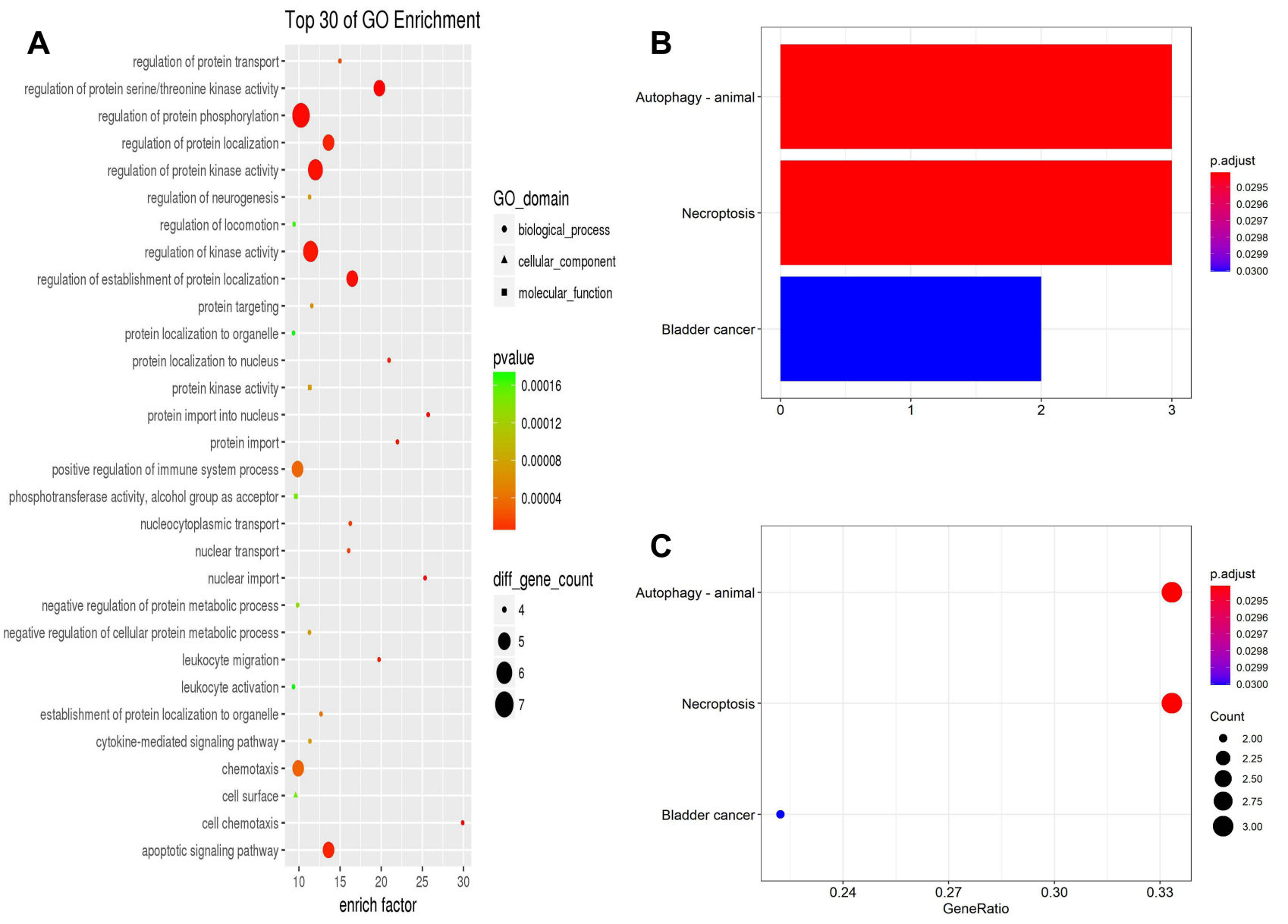

Figure 9 GO (A) analysis shows the biological processes, cellular components, and molecular functions involved in DEARGs. Bar plot (B) and dot plot (C) show KEGG pathway enrichment of DEARGs.

Abbreviations: GO, Gene Ontology; DEARGs, differentially expressed autophagy-related genes; KEGG, Kyoto Encyclopedia of Genes and Genomes.

been found in other studies, as demonstrated by Liu et al, the protein expression level of CXCR4 in AF patients was significantly higher than that in patients with sinus rhythm. ${ }^{39}$ Nevertheless, the current research on ARGs and AF is far from enough and needs further exploration.

Table 3 Significant Enriched GO Terms and Pathways of DEARGs

\begin{tabular}{|c|c|c|c|c|c|c|}
\hline & Term & Count & Genes & P-value & Adj. P. Val & $\mathbf{Q}$ value \\
\hline \multicolumn{7}{|l|}{ GO terms } \\
\hline GO:000I932 (BP) & $\begin{array}{l}\text { Regulation of protein } \\
\text { phosphorylation }\end{array}$ & 7 & $\begin{array}{l}\text { HSP90ABI/DIRAS3/PTK6/CDKNIA/ } \\
\text { IFNG/CXCR4/PRKCD }\end{array}$ & 0.000001317 & & 0.0001825 \\
\hline GO:0045859 (BP) & $\begin{array}{l}\text { Regulation of protein } \\
\text { kinase activity }\end{array}$ & 6 & $\begin{array}{l}\text { HSP90ABI/DIRAS3/PTK6/CDKNIA/ } \\
\text { CXCR4/PRKCD }\end{array}$ & 0.000002055 & & 0.0001708 \\
\hline GO:0043549 (BP) & $\begin{array}{l}\text { Regulation of kinase } \\
\text { activity }\end{array}$ & 6 & $\begin{array}{l}\text { HSP90ABI/DIRAS3/PTK6/CDKNIA/ } \\
\text { CXCR4/PRKCD }\end{array}$ & 0.000002858 & & 0.0001485 \\
\hline GO:0097190 (BP) & $\begin{array}{l}\text { Apoptotic signaling } \\
\text { pathway }\end{array}$ & 5 & $\begin{array}{l}\text { DAPK2/TNFSFI0/CDKNIA/IFNG/ } \\
\text { PRKCD }\end{array}$ & 0.000005167 & & 0.0001464 \\
\hline \multicolumn{7}{|l|}{ KEGG Pathway } \\
\hline hsa04I40 & Autophagy - animal & 3 & DAPK2/ PRKCD/ TP53INP2 & 0.000376076 & $0.0294|26| 4$ & 0.0220710 \\
\hline hsa04217 & Necroptosis & 3 & HSP90ABI/ IFNG/ TNFSFIO & 0.000582428 & $0.0294 \mid 2614$ & 0.0220710 \\
\hline hsa05219 & Bladder cancer & 2 & CDKNIA/ DAPK2 & 0.000891239 & 0.030005034 & 0.0225155 \\
\hline
\end{tabular}

Abbreviations: GO, Gene Ontology; DEARGs, differentially expressed autophagy-related genes; BP, biological process; adj. P. Val, adjust P-value. 
Table 4 Summary of Clinicopathological Variables of Cases and Controls

\begin{tabular}{|l|c|c|l|}
\hline Variables & AF $\mathbf{( N = 1 0 )}$ & Controls $(\mathbf{N}=10)$ & P-value \\
\hline Age (years) & $50 \pm 9.73$ & $45.5 \pm 12.73$ & 0.386 \\
Gender (male/female) & $6 / 4$ & $7 / 3$ & 0.549 \\
Height (cm) & $165.1 \pm 9.267$ & $165 \pm 8.524$ & 0.980 \\
Weight (kg) & $61.55 \pm 12.61$ & $65.2 \pm 12.5 \mathrm{I}$ & 0.524 \\
Body mass index $\left(\mathrm{kg} / \mathrm{m}^{2}\right)$ & $22.34 \pm 2.409$ & $23.76 \pm 2.90 \mathrm{I}$ & 0.249 \\
Hypertension & 2 & 3 & 0.227 \\
Hyperlipidemia & 3 & 7 & 0.179 \\
Smokers & 3 & 5 & 0.774 \\
NYHA functional class & & & 0.631 \\
I & 0 & 1 & \\
II & 4 & 4 & \\
III & 5 & 4 & \\
IV & $\mathrm{I}$ & $\mathrm{I}$ & \\
\hline
\end{tabular}

Notes: Data are presented as mean \pm SD. P-values were calculated using chi-square test, rank sum test, or Student's $t$-test.

Abbreviations: $\mathrm{N}$, number; $\mathrm{SD}$, standard deviation.

In the current study, we discussed 11 potential crucial DEARGs involved in the occurrence and development of $\mathrm{AF}$, suggesting that these genes may serve as potential

A

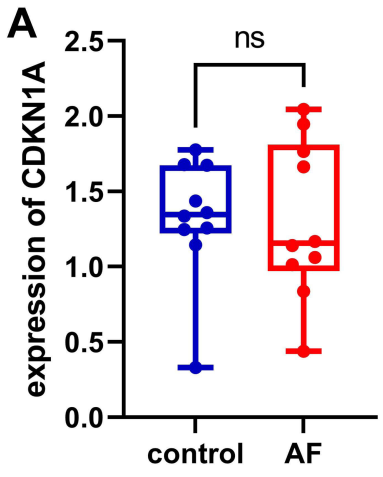

E

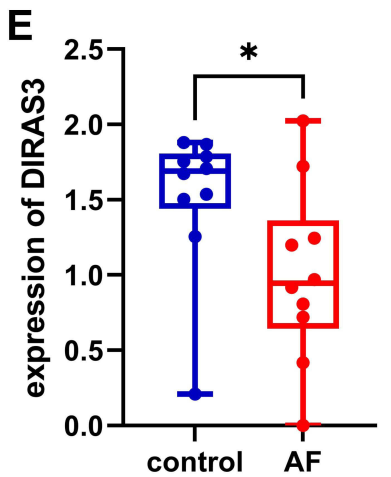

B

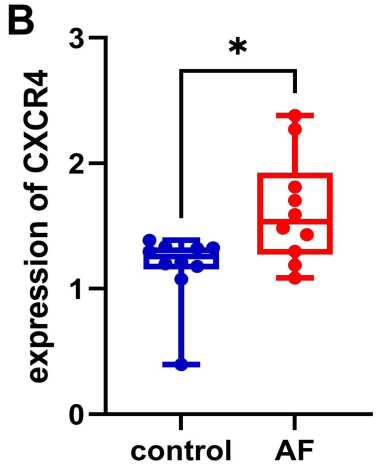

$\mathbf{F}$

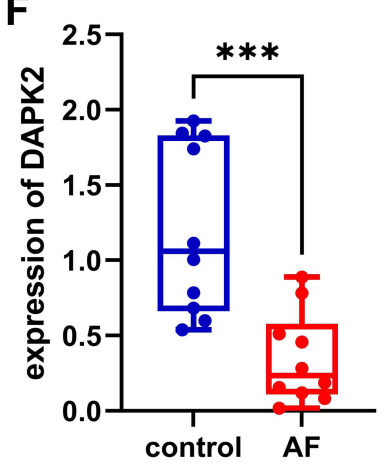

biomarkers and therapeutic targets for AF. However, there are still some limitations in this study. Firstly, due to the small sample size of this study and the heterogeneity of AF, the interpretation of the study results needs to be cautious. In addition, the specific pathophysiological mechanisms of ARGs regulating the initiation and progression of AF need to be further studied. Finally, the working mechanism of these genes is not yet fully understood, so more evidence is needed to discover its biological basis.

\section{Conclusion}

In summary, we conducted the gene differential expression analysis, functional enrichment analysis, and proteinprotein-interaction analysis of autophagy genes in $\mathrm{AF}$, these results show that these DEARGs have great potential as biomarkers and therapeutic targets in AF. Future studies need to further validate the protein interactions identified by RT-PCR. In addition, the crucial genes CXCR4, DAPK2, and TNFSF10 may provide new possibilities for further identifying the susceptibility of $\mathrm{AF}$ and finding useful therapeutic targets.
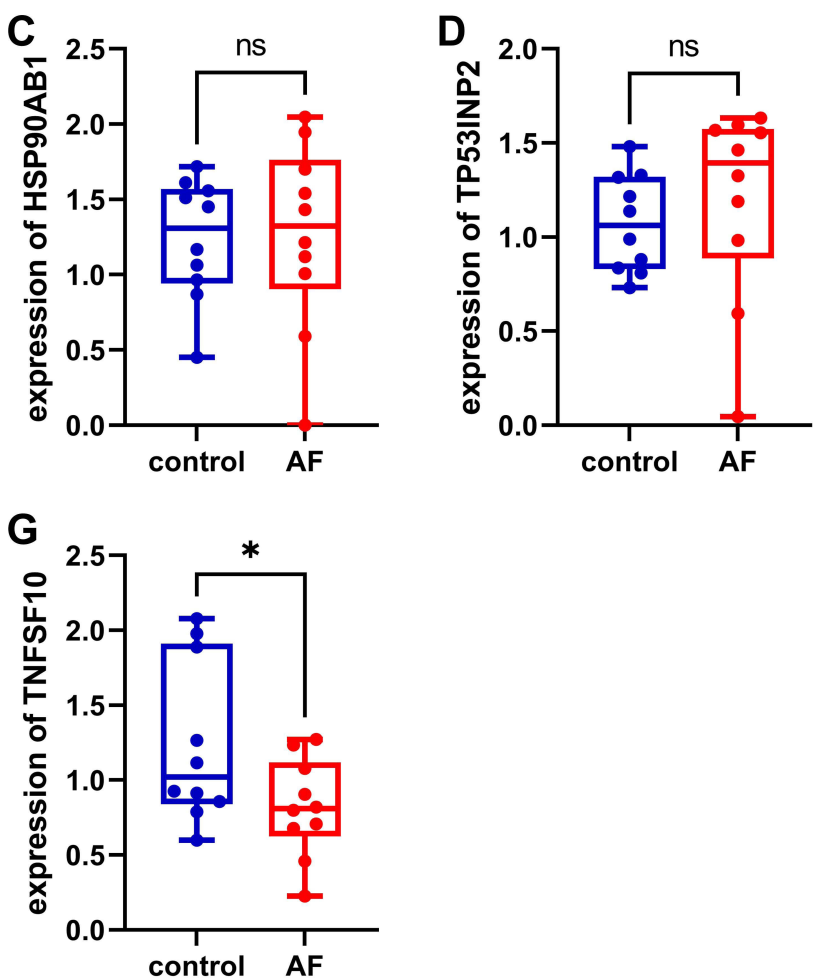

Figure 10 The mRNA expression levels of II DEARGs were measured in AF and non-AF samples. (A) CDKNIA, (B) CXCR4, (C) HSP90ABI, (D) TP53INP2, (E) DIRAS3, (F) DAPK2, (G) TNFSFI0. ${ }^{\mathrm{P}}<0.05$; $* * * \mathrm{P}<0.001$.

Abbreviation: ns, non significant. 


\section{Abbreviations}

AF, atrial fibrillation; ARGs, autophagy-related genes; GEO, Gene Expression Omnibus; DEGs, differentially expressed genes; PPI, protein-protein interaction; DEARGs, differentially expressed ARGs; HADb, Human Autophagy-dedicated Database; GO, Gene Ontology; KEGG, Kyoto Encyclopedia of Genes and Genomes; qRTPCR, quantitative real-time PCR; BP, biological process; BMI, body mass index; $\log 2 \mathrm{FC}, \log 2$ (fold change); AveExpr, average expression; adj. P. Val, adjust P-value.

\section{Data Sharing Statement}

The data analyzed in the present study can be accessed on the GEO (https://www.ncbi.nlm.nih.gov/geo/) website.

\section{Ethics Approval and Informed Consent}

The study protocol was approved by the Ethics Committee of Fuwai Yunnan Cardiovascular Hospital (Ethical Application Ref: IRB2021-BG-006), Kunming, Yunnan, China. Informed consent forms were signed voluntarily by all individual participants included in the study.

\section{Acknowledgments}

We all authors sincerely acknowledge the contribution from the GEO depository.

\section{Funding}

This work was supported by the grant from Yunnan Provincial Cardiovascular Disease Clinical Medical Center Project (No. FZX2019-06-01).

\section{Disclosure}

The authors report no conflicts of interest in this work.

\section{References}

1. Joseph PG, Healey JS, Raina P, et al. Global variations in the prevalence, treatment, and impact of atrial fibrillation in a multi-national cohort of 153152 middle-aged individuals. Cardiovasc Res. 2021;117 (6):1523-1531. doi:10.1093/cvr/cvaa241

2. Yu RB, Li K, Wang G, Gao GM, Du JX. MiR-23 enhances cardiac fibroblast proliferation and suppresses fibroblast apoptosis via targeting TGF- $\beta 1$ in atrial fibrillation. Eur Rev Med Pharmacol Sci. 2019;23 (10):4419-4424.

3. Hu YF, Chen YJ, Lin YJ, Chen SA. Inflammation and the pathogenesis of atrial fibrillation. Nat Rev Cardiol. 2015;12(4):230-243. doi:10.10 38/nrcardio.2015.2

4. Yuan Y, Zhao J, Yan S, et al. Autophagy: a potential novel mechanistic contributor to atrial fibrillation. Int J Cardiol. 2014;172(2):492-494. doi:10.1016/j.ijcard.2014.01.027
5. Liu F, Fang F, Yuan H, et al. Suppression of autophagy by FIP200 deletion leads to osteopenia in mice through the inhibition of osteoblast terminal differentiation. $J$ Bone Miner Res. 2013;28 (11):2414-2430. doi:10.1002/jbmr.1971

6. Galluzzi L, Bravo-San Pedro JM, Levine B, Green DR, Kroemer G. Pharmacological modulation of autophagy: therapeutic potential and persisting obstacles. Nat Rev Drug Discov. 2017;16(7):487-511.

7. Bravo-San Pedro JM, Kroemer G, Galluzzi L. Autophagy and Mitophagy in Cardiovascular Disease. Circ Res. 2017;120 (11):1812-1824. doi:10.1161/CIRCRESAHA.117.311082

8. Ren J, Zhang Y. Targeting autophagy in aging and aging-related cardiovascular diseases. Trends Pharmacol Sci. 2018;39 (12):1064-1076. doi:10.1016/j.tips.2018.10.005

9. Ren J, Sowers JR, Zhang Y. Metabolic stress, autophagy, and cardiovascular aging: from pathophysiology to therapeutics. Trends Endocrinol Metab. 2018;29(10):699-711. doi:10.1016/j.tem.2018. 08.001

10. Mialet-Perez J, Vindis C. Autophagy in health and disease: focus on the cardiovascular system. Essays Biochem. 2017;61(6):721-732. doi:10.1042/EBC20170022

11. Dong Y, Chen H, Gao J, Liu Y, Li J, Wang J. Molecular machinery and interplay of apoptosis and autophagy in coronary heart disease. $J \mathrm{Mol}$ Cell Cardiol. 2019;136:27-41. doi:10.1016/j.yjmcc.2019.09.001

12. Wiersma M, Meijering RAM, Qi XY, et al. Endoplasmic reticulum stress is associated with autophagy and cardiomyocyte remodeling in experimental and human atrial fibrillation. J Am Heart Assoc. 2017;6 (10):e006458. doi:10.1161/JAHA.117.006458

13. Lin $\mathrm{R}, \mathrm{Wu} \mathrm{S}$, Zhu D, Qin M, Liu X. Osteopontin induces atrial fibrosis by activating Akt/GSK-3 $\beta / \beta$-catenin pathway and suppressing autophagy. Life Sci. 2020;245:117328. doi:10.1016/j.lfs.20 20.117328

14. Shingu Y, Takada S, Yokota T, et al. Correlation between increased atrial expression of genes related to fatty acid metabolism and autophagy in patients with chronic atrial fibrillation. PLoS One. 2020;15 (4):e0224713. doi:10.1371/journal.pone.0224713

15. Garcia L, Verdejo HE, Kuzmicic J, et al. Impaired cardiac autophagy in patients developing postoperative atrial fibrillation. J Thorac Cardiovasc Surg. 2012;143(2):451-459. doi:10.1016/j.jtcvs.2011.07.056

16. Yuan Y, Zhao J, Gong Y, et al. Autophagy exacerbates electrical remodeling in atrial fibrillation by ubiquitin-dependent degradation of L-type calcium channel. Cell Death Dis. 2018;9(9):873. doi:10.1038/s41419-018-0860-y

17. Liu A, Jia K, Liang H, Jin Q. Comprehensive analysis of autophagy-related genes and patterns of immune cell infiltration in valvular atrial fibrillation. BMC Cardiovasc Disord. 2021;21(1):132. doi:10.1186/s12872-021-01939-1

18. Barrett T, Wilhite SE, Ledoux P, et al. NCBI GEO: archive for functional genomics data sets-update. Nucleic Acids Res. 2013;41 (Database issue):D991-D995. doi:10.1093/nar/gks1193

19. Szklarczyk D, Gable AL, Lyon D, et al. STRING v11: protein-protein association networks with increased coverage, supporting functional discovery in genome-wide experimental datasets. Nucleic Acids Res. 2019;47(D1):D607-D613. doi:10.1093/nar/gky1131

20. Yu G, Wang LG, Han Y, He QY. clusterProfiler: an R package for comparing biological themes among gene clusters. OMICS. 2012;16 (5):284-287. doi:10.1089/omi.2011.0118

21. Kanehisa M, Goto S. KEGG: Kyoto encyclopedia of genes and genomes. Nucleic Acids Res. 2000;28(1):27-30. doi:10.1093/nar/ 28.1.27

22. Xu J, Cui G, Esmailian F, et al. Atrial extracellular matrix remodeling and the maintenance of atrial fibrillation. Circulation. 2004;109 (3):363-368. doi:10.1161/01.CIR.0000109495.02213.52

23. Wang L, Yuan D, Zheng J, et al. Chikusetsu saponin IVa attenuates isoprenaline-induced myocardial fibrosis in mice through activation autophagy mediated by AMPK/mTOR/ULK1 signaling. Phytomedicine. 2019;58:152764. doi:10.1016/j.phymed.2018.11.024 
24. Lu C, Yang Y, Zhu Y, Lv S, Zhang J. An Intervention Target for Myocardial Fibrosis: autophagy. Biomed Res Int. 2018;2018:6215916. doi:10.1155/2018/6215916

25. Lv S, Yuan P, Dong J, et al. QiShenYiQi pill improves the reparative myocardial fibrosis by regulating autophagy. J Cell Mol Med. 2020;24(19):11283-11293. doi:10.1111/jcmm.15695

26. Liu W, Sun J, Guo Y, et al. Calhex 231 ameliorates myocardial fibrosis post myocardial infarction in rats through the autophagy-NLRP3 inflammasome pathway in macrophages. $J$ Cell Mol Med. 2020;24(22):13440-13453. doi:10.1111/jcmm.15969

27. Hu J, Wang X, Cui X, Kuang W, Li D, Wang J. Quercetin prevents isoprenaline-induced myocardial fibrosis by promoting autophagy via regulating miR-223-3p/FOXO3. Cell Cycle. 2021;20(13):1253-1269. doi:10.1080/15384101.2021.1932029

28. Wang XX, Zhang FR, Zhu JH, Xie XD, Chen JZ. Up-regulation of CXC chemokine receptor 4 expression in chronic atrial fibrillation patients with mitral valve disease may be attenuated by renin-angiotensin system blockers. J Int Med Res. 2009;37 (4):1145-1151. doi:10.1177/147323000903700419

29. Ham SW, Jeon HY, Jin X, et al. TP53 gain-of-function mutation promotes inflammation in glioblastoma. Cell Death Differ. 2019;26 (3):409-425. doi:10.1038/s41418-018-0126-3

30. Dai M, Boudreault J, Wang N, et al. Differential Regulation of Cancer Progression by CDK4/6 Plays a Central Role in DNA Replication and Repair Pathways. Cancer Res. 2021;81 (5):1332-1346. doi:10.1158/0008-5472.CAN-20-2121

31. Xu X, Shen X, Feng W, et al. D-galactose induces senescence of glioblastoma cells through YAP-CDK6 pathway [published online ahead of print, 2020 Sep 29]. Aging. 2020;12(18):18501-18521. doi:10.18632/aging.103819

32. Liu K, Graves JD, Lee YJ, Lin FT, Lin WC. Cell Cycle-Dependent Switch of TopBP1 Functions by Cdk2 and Akt. Mol Cell Biol. 2020;40(8):e00599-19. doi:10.1128/MCB.00599-19
33. Chen SN, Lombardi R, Karmouch J, et al. DNA Damage Response/ TP53 Pathway Is Activated and Contributes to the Pathogenesis of Dilated Cardiomyopathy Associated With LMNA (Lamin A/C) Mutations. Circ Res. 2019;124(6):856-873. doi:10.1161/ CIRCRESAHA.118.314238

34. Haywood ME, Cocciolo A, Porter KF, et al. Transcriptome signature of ventricular arrhythmia in dilated cardiomyopathy reveals increased fibrosis and activated TP53. J Mol Cell Cardiol. 2020;139:124-134. doi:10.1016/j.yjmcc.2019.12.010

35. Birnhuber A, Egemnazarov B, Biasin V, et al. CDK4/6 inhibition enhances pulmonary inflammatory infiltration in bleomycin-induced lung fibrosis. Respir Res. 2020;21(1):167. doi:10.1186/s12931-02001433-w

36. Valkov N, King ME, Moeller J, Liu H, Li X, Zhang P. MicroRNA1-Mediated Inhibition of Cardiac Fibroblast Proliferation Through Targeting Cyclin D2 and CDK6. Front Cardiovasc Med. 2019;6:65. doi:10.3389/fcvm.2019.00065

37. Qi H, Liu Y, Li S, et al. Activation of AMPK Attenuated Cardiac Fibrosis by Inhibiting CDK2 via p21/p27 and miR-29 Family Pathways in Rats. Mol Ther Nucleic Acids. 2017;8:277-290.

38. Shapiro SD, Ranjan AK, Kawase Y, et al. Cyclin A2 induces cardiac regeneration after myocardial infarction through cytokinesis of adult cardiomyocytes. Sci Transl Med. 2014;6(224):224ra27. doi:10.1126/ scitranslmed.3007668

39. Liu P, Sun H, Zhou X, et al. CXCL12/CXCR4 axis as a key mediator in atrial fibrillation via bioinformatics analysis and functional identification. Cell Death Dis. 2021;12(9):813. doi:10.1038/s41419021-04109-5

40. Chu PY, Joshi MS, Horlock D, Kiriazis H, Kaye DM. CXCR4 Antagonism Reduces Cardiac Fibrosis and Improves Cardiac Performance in Dilated Cardiomyopathy. Front Pharmacol. 2019;10:117. doi:10.3389/fphar.2019.00117
International Journal of General Medicine

\section{Publish your work in this journal}

The International Journal of General Medicine is an international, peer-reviewed open-access journal that focuses on general and internal medicine, pathogenesis, epidemiology, diagnosis, monitoring and treatment protocols. The journal is characterized by the rapid reporting of reviews, original research and clinical studies across all disease areas. The manuscript management system is completely online and includes a very quick and fair peer-review system, which is all easy to use. Visit http://www.dovepress.com/ testimonials.php to read real quotes from published authors. 